\title{
Synthesis of Thienamycin methyl ester from 2-deoxy-D-ribose via Kinugasa reaction
}

\author{
Magdalena Soluch, Barbara Grzeszczyk, Olga Staszewska-Krajewska, Marek Chmielewski and \\ Bartłomiej Furman
}

A novel synthesis of thienamycin is described. The crucial step of the synthesis is based on $\mathrm{Cu}(\mathrm{I})$-mediated Kinugasa cycloaddition/rearrangement cascade reaction between terminal acetylene derived from D-lactic acid and suitable, partially protected, five-membered cyclic nitrone obtained from 2-deoxy-D-ribose. The reaction was performed in the presence of tetramethylguanidine as a base to provide 5,6-trans substituted carbapenam as the main product. Thus obtained carbapenam 11 with $(5 R, 6 S)$ configuration at the azetidinone ring was subsequently subjected to oxidation/deprotection/oxidation reaction sequence to afford the $\beta$-keto ester 20 , which was directly transformed into $\mathbf{N}, \mathrm{O}$-protected methyl ester of thienamycin. The Journal of Antibiotics (2016) 69, 164-168; doi:10.1038/ja.2015.108; published online 28 October 2015

\section{INTRODUCTION}

Carbapenems are important $\beta$-lactam antibiotics which, owing to high antibacterial activity and resistance to $\beta$-lactamase, continually attract the interest of industrial and academic laboratories. ${ }^{1-13}$ Recently we have shown that the Kinugasa reaction between D-lactic acid-derived acetylene $\mathbf{2}$ and five-membered cyclic nitrone $\mathbf{1}$ obtained from 2-deoxy-D-ribose ${ }^{14-17}$ offers an attractive entry into carbapenems. ${ }^{18}$ The main drawback of this strategy has been related to the undesired cis configuration of H-5 and H-6 protons in the major adducts 4 or 6 (Scheme 1). We have demonstrated that the stereogenic center at C-6 of the alcohol 4 can be epimerized in the presence of 2 equiv. KHMDS to provide the trans isomer 5 in $55 \%$ yield (Scheme 1). Epimerization performed on compound with protected side-chain hydroxyl 6 caused $\beta$-elimination to the exo double bond.

As effective cis/trans epimerization requires a free hydroxyl group in the side chain and seeing that Kinugasa reaction involving commercially available alcohol 2 and its $t$-butyldiphenysilyl-protected derivative $3^{19}$ was low yielding, we decided to change the side-chain hydroxyl group protection and reaction conditions. After conducting several experiments we found that the protection of alcohol 2 as $t$-butyldimethylsilyl derivative $\mathbf{8}^{20}$ and the use of 1,1,3,3-tetramethylguanidine as the base offered better yield and higher content of the trans isomer 10 which allowed for its easy separation (Scheme 1). This observation was important for the further synthesis of thienamycin because one of the structural features present in carbapenem antibiotics is the trans configuration in the fourmembered $\beta$-lactam ring.

\section{RESULTS AND DISCUSION}

Selective debenzylation of the primary hydroxyl group ${ }^{21}$ in $\mathbf{1 0}$ by hydrogenolysis under the pressure of 6 bar $\mathrm{H}_{2}$ provided a mixture of two regioisomers 11 and 12 (6:1), however, in a low yield (35\%) only. Higher pressure led to debenzylation of both groups to afford $\mathbf{1 3}$ in low yield. The same deprotection of both benzyl groups can be achieved by a reduction with sodium in liquid ammonia (Figure 1). ${ }^{22}$

This results prompted us to use partly deprotected nitrone 14 in the Kinugasa reaction with $\mathbf{8}$. Nitrone 14 was obtained from 1 by a modification of a known method, ${ }^{23,24}$ using the complex of $\mathrm{BCl}_{3}$ with dimethyl sulfide. Under such milder conditions, only the primary hydroxyl group was deprotected in 50\% yield (Scheme 2 ).

The reaction of $\mathbf{1 4}$ with $\mathbf{8}$ gave a mixture of cis and trans carbapenams 15 and 11 in a ratio of about 1:3 and with $54 \%$ overall yield. The trans adduct 11 was separated by chromatography. The primary hydroxyl group was oxidized to the aldehyde using DessMartin periodinane $e^{25}$ in $91 \%$ yield and subsequently the crude product was oxidized with sodium chlorite under PinnickLindgren ${ }^{26,27}$ conditions to afford the carboxylic acid function, which was in turn methylated with diazomethane ${ }^{28}$ to afford ester 18 (Scheme 3).

The benzyl protecting group was then removed using palladium hydroxide on carbon to afford the alcohol 19 in 97\% yield. The structure and configuration of alcohol 19 was confirmed by X-ray crystallography. Compound 19 was then oxidized to ketone 20 using Dess-Martin reagent (Scheme 4). It's important to know that the similar keto-p-nitrobenzyl ester 20a has already been transformed into thienamycin by the Hannesian group. ${ }^{29}$

The transformation of the ketone $\mathbf{2 0}$ into thienamycin derivative $\mathbf{2 1}$ according to a known procedure required the enolization of the carbonyl group followed by phosphorylation of the enol hydroxyl and subsequent addition of $\mathrm{N}$-acetylcysteamine. ${ }^{29}$ The best result was obtained for diethyl chlorophosphate as a phosphorylation agent. We also found that the enolization of ketone $\mathbf{2 0}$ required quite a long time. 


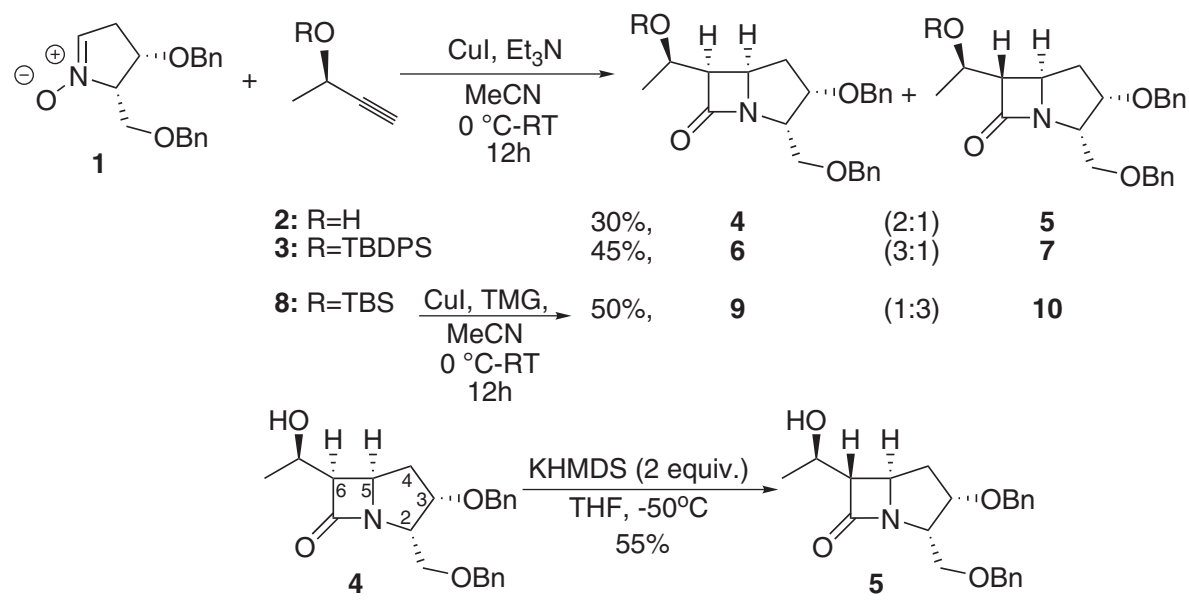

Scheme 1 Synthesis of carbapenams via Kinugasa reaction with $\mathrm{Et}_{3} \mathrm{~N}$ and 1,1,3,3-tetramethylguanidine as a base. Attempted epimerization at C-6.

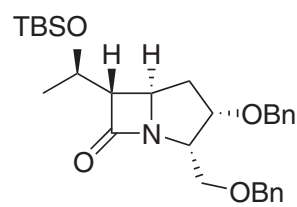

10

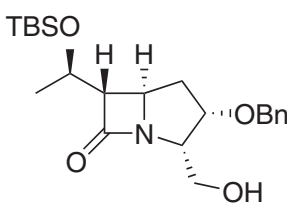

11

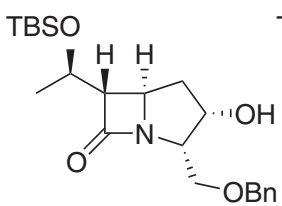

12<smiles>CC(O[SnH3])[C@H]1C(=O)N2[C@@H](CO)[C@@H](O)C[C@H]12</smiles>

13

Figure 1 Selective debenzylation of the primary hydroxyl group.

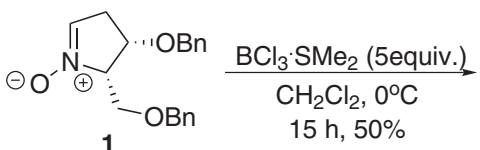<smiles>O=[N+]([O-])[C@@H]1CC[C@@H](OBr)[C@@H]1O</smiles><smiles>CC(O[SnH3])C1C(=O)N2C1C[C@@H](Br)C2CO</smiles>

15

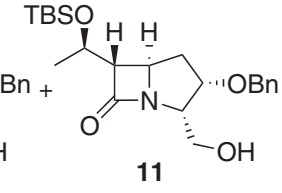

Scheme 2 Deprotection of nitrone 1 and Kinugasa reaction with alkyne 8.

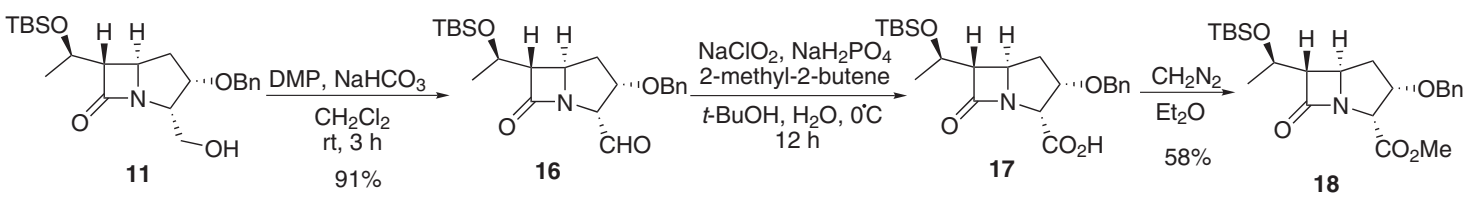

Scheme 3 Preparation of carbapenam 18.

Otherwise phosphorylation did not occur and compound 21 was not formed. Particularly when treating $\mathbf{2 0}$ with diphenyl chlorophosphate, we observed the opening of the pyrrolidine ring via retro-Dieckmann reaction and formation of thioester 22 (Scheme 5).

In conclusion, we have demonstrated that the cyclic five-membered nitrone 1, easily available from 2-deoxy-D-ribose represents an attractive substrate for the stereocontrolled synthesis of thienamycin -an important natural carbapenem antibiotic. The configuration of the stereogenic center next to the nitrogen atom has a decisive role in the asymmetric induction at the C-5 carbon atom of the carbapenam scaffold. The predominance of the isomer with anti configuration of $\mathrm{H}-5$ and $\mathrm{H}-6$ protons was achieved by the use of tetramethylguanidine as a base in the Kinugasa reaction.

\section{EXPERIMENTAL PROCEDURE}

Melting points were determined using Köfler hot-stage apparatus with microscope and were uncorrected. Proton and carbon NMR spectra were recorded on a Varian VN MRS Spectrometer (Varian Inc., St Clara, CA, USA) at 600 and $150 \mathrm{MHz}$, respectively, in $\mathrm{CDCl}_{3}$ or $\mathrm{C}_{6} \mathrm{D}_{6}$. IR spectra were obtained on an FT-IR-1600 Perkin-Elmer spectrophotometer (Perkin Elmer, Waltham, MA, USA). The optical rotations were measured with a JASCO J-2000 digital polarimeter (Jasco Inc., Easton, MD, USA). High-resolution mass spectra were recorded on ESI-TOF Mariner spectrometer (Perspective Biosystem, Framingham, MA, USA). X-ray analysis were performed on Nonius MACH3 diffractometer (Bruker, Madison, WI, USA). The HPLC analysis were carried out on Hitachi chromatograph (Hitaschi, Tokyo, Japan) with L-2130 pump and L-2450 DAD detector (Brucker, Karlsruhe, Germany) equipped with LiChrospher Si60 analytical column (Merck, Darmstadt, Germany). 


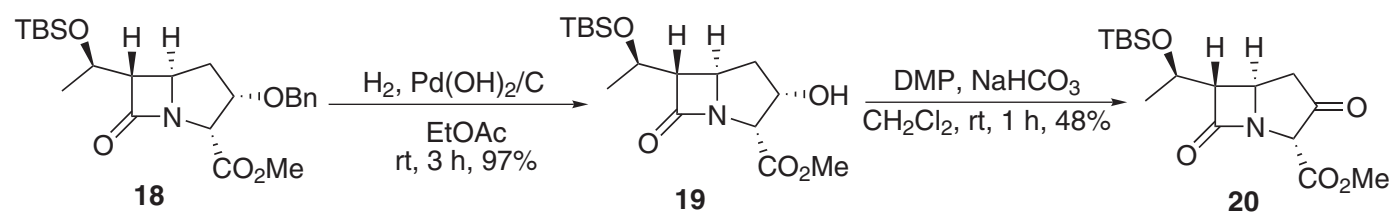<smiles>CC(C)OC(C)[C@H]1C(=O)N2[C@@H](C(=O)OCc3ccc([N+](=O)[O-])cc3)C(=O)C[C@H]12</smiles>

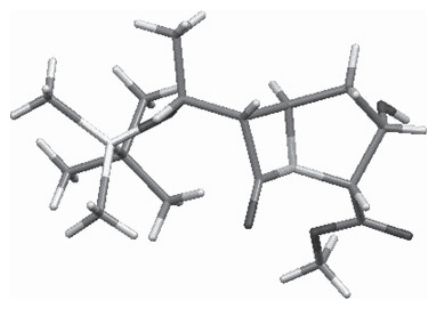

X-ray structure of compound 19

Scheme 4 Synthesis of ketone 20. A full color version of this scheme is available at The Journal of Antibiotics journal online.

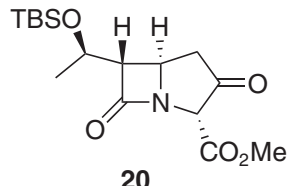

20

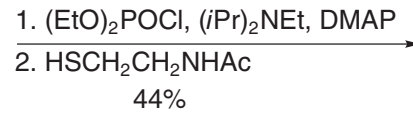

. (EtO) ${ }_{2} \mathrm{POCl},(\mathrm{Pr})_{2} \mathrm{NEt}, \mathrm{DMAP}$

$44 \%$

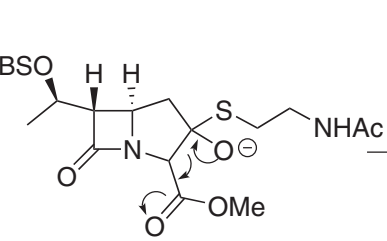

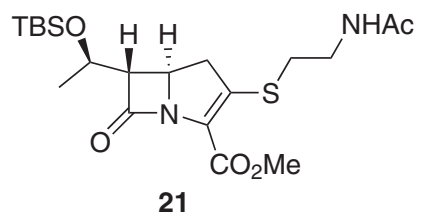

21

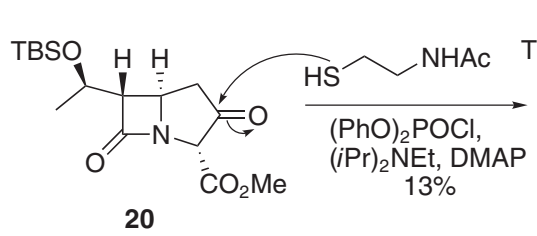<smiles>COC(=O)C1=C(SCCNC(C)(C)C)C[C@@H]2[C@@H](C(C)OC(C)(C)C)C(=O)N12</smiles>

21<smiles>COC(=O)CN1C(=O)[C@H](C(C)O[R15](C)(F)F)[C@H]1CC(=O)SCCN</smiles>

Scheme 5 Synthesis of protected thienamycin $\mathbf{2 1}$ and side reactions in the phosphorylation-addition sequence.

Thin-layer chromatography (TLC) was performed on aluminum sheets silica gel $60 \mathrm{~F}_{254}$ from Merck. Column chromatography (CC) was carried out using Merck silica gel (230-400 mesh) or Florisil (100-200 mesh). The TLC spots were visualized in UV $(254 \mathrm{~nm})$ and by treatment with alcoholic solution of ninhydrine, aqueous solution of $\mathrm{KMnO}_{4}$ or with ceric sulphate/phosphomolybdic acid solution.

All solvents were dried and purified applying standard techniques.

\section{(2S,3S)-3-(benzyloxy)-2-((benzyloxy)methyl)-3,4-dihydro- $2 \mathrm{H}$ - pyrrole-1-oxide (1) Compound 1 was prepared according to literature methods}

Step 1: To a solution of 3,5-di-O-benzyl-2-deoxy-D-ribofuranoside ${ }^{15}$ $(8.91 \mathrm{~g}, 28.3 \mathrm{mmol})^{16}$ in dry toluene $(70 \mathrm{ml})$ was added $\mathrm{MgSO}_{4}(10.3 \mathrm{~g})$ under argon. The suspension was stirred at reflux temperature for $5 \mathrm{~min}$, and then O-tert-butyldiphenylsilylhydroxylamine $(8.6 \mathrm{~g}, 32 \mathrm{mmol})$ and pyridinium $p$-toluenesulfonate $(98 \mathrm{mg}$ ) were added. The reaction mixture was heated at the same temperature for $30 \mathrm{~min}$, and then it was filtered. The filtrate was washed with a saturated aqueous solution of $\mathrm{NaHCO}_{3}$ and with brine and dried over $\mathrm{MgSO}_{4}$ to give a residue, which was use in the next step without further purification.

Step 2: To a solution of oxime with previous step $(12.0 \mathrm{~g}, 21.2 \mathrm{mmol})$ in $\mathrm{CH}_{2} \mathrm{Cl}_{2}(70 \mathrm{ml})$ placed at $0{ }^{\circ} \mathrm{C}$ under argon atmosphere were added successively triethylamine $(4.5 \mathrm{ml}, 32.8 \mathrm{mmol})$ and mesyl chloride $(1.9 \mathrm{ml}, 24.6 \mathrm{mmol})$. The solution was stirred for $30 \mathrm{~min}$, and then water $(15 \mathrm{ml})$ was added. The aqueous layer was extracted three times with $\mathrm{CH}_{2} \mathrm{Cl}_{2}$. The organic layers were washed with brine, dried over $\mathrm{MgSO}_{4}$, and concentrated under vacuum to give a residue, which upon short column chromatography over silica gel (pentane/ AcOEt 9/1, 4/1, then 1/1) yielded the desired unstable mesylate (13.4 g, 97.6\%:
3/1 mixture of $E / Z$ isomers) as an yellow oil. To a solution of mesylate (13.4 g, $20.7 \mathrm{mmol})$ in THF $(400 \mathrm{ml})$ placed at $0{ }^{\circ} \mathrm{C}$ under argon was added tetrabutylammonium fluoride $(24.2 \mathrm{ml}, 1 \mathrm{~m}$ solution in THF, $24.2 \mathrm{mmol})$. The reaction mixture was stirred for $5 \mathrm{~min}$ at $0^{\circ} \mathrm{C}$, and then the solvent was removed under vacuum. The residue was dissolved in ethyl acetate, water was added, and the aqueous layer was extracted three times with ethyl acetate. The organic layers were washed with brine, dried over $\mathrm{MgSO}_{4}$, and concentrated under vacuum to give a residue, which was use in the next step without further purification.

Step 3: To a solution of crude oxime with previous step (6.2 g, $18.8 \mathrm{mmol})$ in a $4: 1$ mixture of methanol and water $(200 \mathrm{ml})$ were added $\mathrm{NaHCO}_{3}(8.5 \mathrm{~g}$, $102.0 \mathrm{mmol})$ and hydroxylamine hydrochloride $(6.7 \mathrm{~g}, 96.5 \mathrm{mmol})$. The reaction mixture was heated overnight at reflux temperature. After concentration under vacuum, the residue was dissolved in $\mathrm{CH}_{2} \mathrm{Cl}_{2}$, and water was added. The aqueous layer was extracted three times with $\mathrm{CH}_{2} \mathrm{Cl}_{2}$, and the organic layers were washed with brine, dried over $\mathrm{MgSO}_{4}$ and concentrated under vacuum to give a residue, which upon column chromatography over silica gel (acetone/ DCM 1/9 then methanol/DCM 5/95) yielded the nitrone 1 (5.1 g, 87\%) as an olive syrup.

$[\alpha]_{\mathrm{D}}+23,7\left(c 1, \mathrm{CHCl}_{3}\right)$, IR (film) v: 737, 1113, 2866, 3062, $3239 \mathrm{~cm}^{-1}$

${ }^{1} \mathrm{H}$ NMR $\left(600 \mathrm{MHz}, \mathrm{CDCl}_{3}\right) \delta: 2.74-2.78(\mathrm{~m}, 1 \mathrm{H}, \mathrm{H}-3), 2.79-2.85$ (ddd $J=2.6 \mathrm{~Hz}, 7,1 \mathrm{~Hz}, 17,9 \mathrm{~Hz}, 1 \mathrm{H}, \mathrm{H}-3), 4.00-4.05(\mathrm{~m}, 1 \mathrm{H}, \mathrm{H}-5), 4.07-4.12$ (m, $\left.2 \mathrm{H}, \mathrm{CH}_{2} \mathrm{OBn}\right), 4.45-4.50(\mathrm{~m}, 1 \mathrm{H}, \mathrm{H}-4), 4.54-4.62\left(\mathrm{~m}, 2 \mathrm{H}, 2 \times \mathrm{CH}_{2} \mathrm{Ph}\right)$, 6.85-6.88 (m, 1H, H-2). ${ }^{13} \mathrm{C}$ NMR $\left(150 \mathrm{MHz}, \mathrm{CDCl}_{3}\right) \delta: 35.03,64.86,72.20$, $73.63,73.66,74.32,127.56,127.60,127.66,127.94,128.31,128.50,133.23$, 137.41, 137.99 .

HR MS (ESI): m/z [M+Na] ${ }^{+}$calculated. for $\mathrm{C}_{19} \mathrm{H}_{21} \mathrm{NO}_{3} \mathrm{Na}$ : 334,1419; found: 334,1427 
$(2 S, 3 S)$-3-Benzyloxy-2-hydroxymethyl-3,4-dihydro- $2 \mathrm{H}$-pyrrole- $\mathrm{N}$ oxide (14)

To a solution of nitrone $1(0.46 \mathrm{~g}, 1.50 \mathrm{mmol})$ in $\mathrm{CH}_{2} \mathrm{Cl}_{2}(30 \mathrm{ml})$, cooled to $0{ }^{\circ} \mathrm{C}, \mathrm{BCl}_{3} \bullet \mathrm{Me}_{2} \mathrm{~S}$ complex $(1 \mathrm{ml}, 7.50 \mathrm{mmol})$ was added. The reaction mixture was stirred at room temperature overnight. Subsequently the mixture was evaporated, treated with methanol and evaporated again $(4 \times 10 \mathrm{ml})$. The crude mixture was purified by silica gel chromatography to afford the nitrone $\mathbf{1 4}$ $(0.17 \mathrm{~g}, 0.75 \mathrm{mmol})$ in $50 \%$ yield.

Colorless syrup; $[\alpha]_{\mathrm{D}}+23,7$ (c 1, $\mathrm{CHCl}_{3}$ ), IR (film) v: 1453, 2925, 3030, $3334 \mathrm{~cm}^{-1}$

${ }^{1} \mathrm{H}$ NMR $\left(600 \mathrm{MHz}, \mathrm{CDCl}_{3}\right) \delta: 2.70-2.77(\mathrm{~m}, 1 \mathrm{H}, \mathrm{H}-4), 2.85-2.94(\mathrm{~m}, 1 \mathrm{H}$, $\mathrm{H}-4), 4.01-4.07(\mathrm{~m}, 1 \mathrm{H}, \mathrm{CHHOH}), 4.09-4.15(\mathrm{~m}, 2 \mathrm{H}, \mathrm{H}-2, \mathrm{OH}), 4.20-4.45$ $(\mathrm{m}, 1 \mathrm{H}, \mathrm{CH} H \mathrm{OH}), 4.43-4.62\left(\mathrm{~m}, 3 \mathrm{H}, \mathrm{CH}_{2} \mathrm{Ph}, \mathrm{H}-3\right), 6.89$ (s, 1H, H-5), 7.24-7.40 (m, 5H, Ar). ${ }^{13} \mathrm{C}$ NMR $\left(150 \mathrm{MHz}, \mathrm{CDCl}_{3}\right) \delta: 34.93,60.35,71.78$, $73.53,76.79,127.60,128.26,128.52,128.68,136.72$.

HR MS (ESI): m/z [M+Na] $]^{+}$calculated for $\mathrm{C}_{12} \mathrm{H}_{15} \mathrm{NO}_{3} \mathrm{Na}$ : 244,1086; found: 244,1088

(2S,3S,5R,6S,1'R)-3-Benzyloxy-6-[1'-(tert-butyldimethylosiloxy) ethyl]-2-(hydroxymethyl)-1-azabicyclo[3.2.0]heptan-7-one (15) and (2S,3S,5R,6R,1'R)-3-benzyloxy-6-[1'-(tert-butyldimethylosiloxy) ethyl]-2-(hydroxymethyl)-1-azabicyclo [3.2.0] heptan-7-one (11) To a suspension of $\mathrm{CuI}(0.5 \mathrm{mmol}, 95 \mathrm{mg})$ in dry, degassed $\mathrm{MeCN}(3 \mathrm{ml})$, $253 \mu \mathrm{l}(2.0 \mathrm{mmol})$ of 1,1,3,3-tetramethylguanidine was added. After cooling to $0^{\circ} \mathrm{C}$ a solution of acetylene $8^{20}(92 \mathrm{mg}, 0.5 \mathrm{mmol})$ in $1 \mathrm{ml}$ of MeCN was added and thus obtained mixture was stirred for $15 \mathrm{~min}$. Then a solution of nitrone 14 $(221 \mathrm{mg}, 1.0 \mathrm{mmol})$ in $\mathrm{MeCN}(2 \mathrm{ml})$ was added slowly and the mixture was kept at $0{ }^{\circ} \mathrm{C}$ for additional $15 \mathrm{~min}$. After removal of the cooling bath, the mixture was stirred at ambient temperature under an inert atmosphere for $24 \mathrm{~h}$. The progress of the reaction was monitored by TLC. Removal of the solvent gave a residue, which was chromatographed on silica gel (hexane/AcOEt 4:6 v/ v) to afford a mixture of 15 and 11 in ratio $1: 3\left(110 \mathrm{mg}, 54 \%\right.$, according to ${ }^{1} \mathrm{H}$ NMR of crude reaction mixture).

Compound 11; colorless syrup; $[\alpha]_{\mathrm{D}}+64,3\left(c 1, \mathrm{CHCl}_{3}\right)$; IR (film) v: 1099, $1757,2856,3437 \mathrm{~cm}^{-1} ;{ }^{1} \mathrm{H}$ NMR $\left(600 \mathrm{MHz}, \mathrm{CDCl}_{3}\right) \delta: 0.06\left(\mathrm{~s}, 6 \mathrm{H}, \mathrm{Si}\left(\mathrm{CH}_{3}\right)_{2}\right)$, $0,87(\mathrm{~s}, 9 \mathrm{H}, t-\mathrm{Bu}), 1.22\left(\mathrm{~d}, J=6.1 \mathrm{~Hz}, 3 \mathrm{H}, \mathrm{CH}_{3}\right), 1.68-1.73(\mathrm{~m}, 1 \mathrm{H}, \mathrm{H}-4)$, 2.31-2.36 (m, $1 \mathrm{H}, \mathrm{H}-4), 2.80(\mathrm{dd}, J=2.0 \mathrm{~Hz}, 6,0 \mathrm{~Hz}, 1 \mathrm{H}, \mathrm{H}-6), 3.73$ $\left(\mathrm{d}, J=5.6 \mathrm{~Hz}, 2 \mathrm{H}, \mathrm{CH}_{2} \mathrm{OH}\right), 3,86-3.89(\mathrm{~m}, 1 \mathrm{H}, \mathrm{H}-5), 4.00(\mathrm{q}, 1 \mathrm{H}, J=5.8 \mathrm{~Hz}$, $\mathrm{H}-2), 4,15-4.20(\mathrm{~m}, 1 \mathrm{H}, \mathrm{CHOSi}), 4.44-4.46(\mathrm{~m}, 1 \mathrm{H}, \mathrm{H}-3),, 4.45-4.64(\mathrm{~m}, 2 \mathrm{H}$, $\left.\mathrm{CH}_{2} \mathrm{Ph}\right), 7.28-7.32(\mathrm{~m}, 3 \mathrm{H}, \mathrm{Ar}), 7.34-7.37(\mathrm{~m}, 2 \mathrm{H}, \mathrm{Ar}) ;{ }^{13} \mathrm{C}$ NMR $(150 \mathrm{MHz}$, $\left.\mathrm{CDCl}_{3}\right) \delta:-4.94,-4.22,17,95,22,61,25,68,53.81,61.28,62.65,65.23,66.18$, $72.33,84.95,127.57,128.64,137.33,177.16$; HR MS(ESI) $\mathrm{m} / \mathrm{z}[\mathrm{M}+\mathrm{Na}]^{+}$ calculated for $\mathrm{C}_{22} \mathrm{H}_{35} \mathrm{NO}_{4} \mathrm{SiNa}$ : 428.2233; found: 428.2234 .

Compound 15: colorless syrup; $[\alpha]_{\mathrm{D}}+39,1\left(c 1, \mathrm{CHCl}_{3}\right)$; IR (film) v: 1092, $1744,2926,3400 \mathrm{~cm}^{-1} ;{ }^{1} \mathrm{H}$ NMR $\left(600 \mathrm{MHz}, \mathrm{CDCl}_{3}\right) \delta: 0.06\left(\mathrm{~s}, 6 \mathrm{H}, \mathrm{Si}\left(\mathrm{CH}_{3}\right)_{2}\right)$, $0,88(\mathrm{~s}, 9 \mathrm{H}, t-\mathrm{Bu}), 1.20\left(\mathrm{~d}, J=6.2 \mathrm{~Hz}, 3 \mathrm{H}, \mathrm{CH}_{3}\right), 1.82-1.87(\mathrm{~m}, 1 \mathrm{H}, \mathrm{H}-4)$, 2.15-2.20 (m, 1H, H-4), 3.39(dd, $J=5.7 \mathrm{~Hz}, 7,7 \mathrm{~Hz}, 1 \mathrm{H}, \mathrm{H}-6), 3.71-3.3 .78$ $\left(\mathrm{m}, 2 \mathrm{H}, \mathrm{CH}_{2} \mathrm{OH}\right), 3.90(\mathrm{q}, J=5.7 \mathrm{~Hz}, 1 \mathrm{H}, \mathrm{H}-2), 3.91-3.95(\mathrm{~m}, 1 \mathrm{H}, \mathrm{H}-5)$, $4.00-4.05(\mathrm{~m}, 1 \mathrm{H}, \mathrm{CHSi}), 4.42-4.45(\mathrm{~m}, 1 \mathrm{H}, \mathrm{H}-3), 4.46-4.63\left(\mathrm{~m}, 2 \mathrm{H}, \mathrm{CH}_{2} \mathrm{Ph}\right)$, $7.35-7.37(\mathrm{~m}, 5 \mathrm{H}, \mathrm{Ar}) ;{ }^{13} \mathrm{C}$ NMR $\left(150 \mathrm{MHz}, \mathrm{CDCl}_{3}\right) \delta:-4.55,-4.33,22.84$, $25.81,29.68,32.08,54.20,58.12,61.52,61.94,65.36,72.27,84.66,126.97$, $127.64,128.55,140.87,178.37$; HR MS(ESI) $\mathrm{m} / z[\mathrm{M}+\mathrm{Na}]^{+}$calculated. for $\mathrm{C}_{22} \mathrm{H}_{35} \mathrm{NO}_{4} \mathrm{SiNa}$ : 428.2233; found: 428.2227 .

\section{(2R,3S,5R,6S,1'R)-3-Benzyloxy-6-[1'-(tert-butyldimethylsiloxy)} ethyl]-2-methoxycarbonyl-1-azabicyclo[3.2.0] heptan-7-one (18)

To a solution of $11(166 \mathrm{mg}, 0.4 \mathrm{mmol})$ in $\mathrm{CH}_{2} \mathrm{Cl}_{2}(15 \mathrm{ml})$ sodium bicarbonate $(134 \mathrm{mg}, 1.6 \mathrm{mmol})$ and Dess-Martin periodinane $(340 \mathrm{mg}, 0.8 \mathrm{mmol})$ were added. The reaction mixture was stirred at room temperature for $1 \mathrm{~h}$. Subsequently, a saturated solution of $\mathrm{Na}_{2} \mathrm{~S}_{2} \mathrm{O}_{3}$ was added. The water phase was extracted with $\mathrm{CH}_{2} \mathrm{Cl}_{2}(3 \times)$. The combined extracts were washed with brine and dried over $\mathrm{MgSO}_{4}$. The solution was evaporated and the crude aldehyde $\mathbf{1 6}$ was used in the next step without purification.

The crude $\mathbf{1 6}$ was dissolved in $t$ - $\mathrm{BuOH}(6 \mathrm{ml})$ and treated with 2-methyl-2butene $(6 \mathrm{ml})$. Subsequently $\mathrm{NaClO}_{2}(0.21 \mathrm{~g}, 2.3 \mathrm{mmol})$ and a solution of $\mathrm{NaH}_{2} \mathrm{PO}_{4}(0.26 \mathrm{~g}, 1.90 \mathrm{mmol})$ in water $(11 \mathrm{ml})$ were added. The reaction mixture was stirred overnight at room temperature. $\mathrm{NH}_{4} \mathrm{Cl}$ was added and the aqueous solution was extracted with ethyl acetate (3X). The combined extracts were dried over $\mathrm{MgSO} 4$, filtered and evaporated. The crude acid 17 was dissolved in $\mathrm{Et}_{2} \mathrm{O}(20 \mathrm{ml})$, cooled to $0^{\circ} \mathrm{C}$ and treated with a solution of diazomethane. After 5 min the mixture was treated with drop of acetic acid and evaporated. The product was purified on a silica gel column using hexane-EtOAc, 6:4 v/v as an eluant to give $\mathbf{1 8}$ in $58 \%$ yield.

Colorless syrup; $[\alpha]_{\mathrm{D}}+121,1\left(c 1, \mathrm{CHCl}_{3}\right)$; IR (film) v: 1099, 1746, 1792, $2929, \mathrm{~cm}^{-1} ;{ }^{1} \mathrm{H}$ NMR $\left(500 \mathrm{MHz}, \mathrm{CDCl}_{3}\right) \delta: 0.08\left(\mathrm{~s}, 6 \mathrm{H}, \mathrm{Si}\left(\mathrm{CH}_{3}\right)_{2}\right), 0,89(\mathrm{~s}, 9 \mathrm{H}$, $t$-Bu), $1.24\left(\mathrm{~d}, J=6.0,3 \mathrm{H}, \mathrm{CH}_{3}\right), 1.62-1.69(\mathrm{~m}, 1 \mathrm{H}, \mathrm{H}-4), 2.32-2.38(\mathrm{~m}, 1 \mathrm{H}$, $\mathrm{H}-4$ ), 2.82 (dd, $J=2.1 \mathrm{~Hz}, 6,4 \mathrm{~Hz}, 1 \mathrm{H}, \mathrm{H}-6$ ), 3.70 (s, $3 \mathrm{H}, \mathrm{CO}_{2} \mathrm{CH}_{3}$ ), 4.03-4.08 (m, $1 \mathrm{H}, \mathrm{H}-5), 4.19-4.25(\mathrm{~m}, 1 \mathrm{H}, \mathrm{CHOSi}), 4.55\left(\mathrm{~s}, 2 \mathrm{H}, \mathrm{OCH}_{2} \mathrm{Ph}\right), 4.57-4.61$ $(\mathrm{m}, 1 \mathrm{H}, \mathrm{H}-3), 4.61-4.64(\mathrm{~m}, 1 \mathrm{H}, \mathrm{H}-2), 7.25-7.36(\mathrm{~m}, 5 \mathrm{H}, \mathrm{Ar}) ;{ }^{13} \mathrm{C}$ NMR $\left(150 \mathrm{MHz}, \mathrm{CDCl}_{3}\right) \delta:-4.21,17.93,22.62,25.69,36.02,52.03,55.28,63.70$, $65.34,66.25,72.61,85.47,127.54,127.88,128.42,137.33,168.59,175.88$.

HR MS(ESI) $m / z[\mathrm{M}+\mathrm{Na}]^{+}$calculated. for $\mathrm{C}_{23} \mathrm{H}_{35} \mathrm{NO}_{5} \mathrm{SiNa}$ : 456.2182 ; found: 456.2185 .

$(2 R, 3 S, 5 R, 6 S, 1$ 'R)-6-[1'-(tert-Butyldimethylsiloxy)ethyl]-3-hydroxy2-methoxycarbonyl-1-azabicyclo[3.2.0] heptan-7-one (19)

Compound $18(0.10 \mathrm{~g}, 0,30 \mathrm{mmol})$ in ethyl acetate $(10 \mathrm{ml})$ was treated with Pd $(\mathrm{OH})_{2}(0.15 \mathrm{~g})$ and hydrogenated under $1 \mathrm{~atm} \mathrm{H}_{2}$ at room temp. for $3 \mathrm{~h}$. Subsequently the catalyst was filtered off through a short path of celite. The solution was evaporated and purified on a silica gel column using hexane/ EtOAc 4:6 v/v as an eluent to give $19(0.10 \mathrm{~g}, 0.29 \mathrm{mmol})$ in $97 \%$ yield.

Colorless crystals; $[\alpha]_{\mathrm{D}}+103,8\left(c 1, \mathrm{CHCl}_{3}\right) ;$ m.p. $=151-153^{\circ} \mathrm{C}$;

IR (film) $v: 1718,1735,2927,3337 \mathrm{~cm}^{-1}$;

${ }^{1} \mathrm{H}$ NMR $\left(500 \mathrm{MHz}, \mathrm{CDCl}_{3}\right) \delta: 0.09\left(\mathrm{~s}, 6 \mathrm{H}, \mathrm{Si}\left(\mathrm{CH}_{3}\right)_{2}\right), 0,89$ (s, 9H, $t$-Bu), 1.25 (d, J=6.2 Hz, 3H, $\left.\mathrm{CH}_{3}\right), 1.69-1.70(\mathrm{~m}, 1 \mathrm{H}, \mathrm{H}-4), 2.34-2.36$ (m, $\left.1 \mathrm{H}, \mathrm{H}-4\right)$, 2.69 (bs, $1 \mathrm{H}, \mathrm{OH}), 2.85$ (dd, $J=1.8 \mathrm{~Hz}, 5,8 \mathrm{~Hz}, 1 \mathrm{H}, \mathrm{H}-6), 3.77\left(\mathrm{~s}, 3 \mathrm{H}, \mathrm{CO}_{2} \mathrm{CH}_{3}\right.$ ), 4.09-4.10 (m, 1H, H-5), 4.24-4.26 (m, $1 \mathrm{H}, \mathrm{CHOSi}), 4.5(\mathrm{~d}, J=4.8 \mathrm{~Hz}, 1 \mathrm{H}$, H-2), 4.94-4.95 (m, 1H, H-3);

${ }^{13} \mathrm{C}$ NMR $\left(150 \mathrm{MHz}, \mathrm{CDCl}_{3}\right) \delta:-4.99,-4.22,17.94,22.64,25.67,39.41$, $52.45,55.33,64.56,64.84,66.00,79.06,169.91,176.13$.

HR MS(ESI) $\mathrm{m} / z$ [M+Na] $]^{+}$calculated for $\mathrm{C}_{16} \mathrm{H}_{29} \mathrm{NO}_{5} \mathrm{SiNa}$ : 366.1713; found: 366.1707 .

Crystallographic data: $\mathrm{C}_{16} \mathrm{H}_{29} \mathrm{NO}_{5} \mathrm{Si}, \mathrm{MW}=343.49 \mathrm{Da}, 0.40 \times 0.40 \times 0.05$, monoclinic, space group. $P=21, Z=2, T=100(2) \mathrm{K}, a=7.3250(2)$, $b=7.5160(2), c=17.4905(5) \AA, V=962.89(5) \AA^{3}, \lambda(\mathrm{Cu}, \mathrm{K} \alpha)=1.54184 \AA$, $\mu=1.271 \mathrm{~mm}^{-1}$. Intensity data were collected on SuperNova area detector system. The structure was solved by direct mehods and refined by the fullmatrix least-squares on $F^{2}$ (SHELXL-97). A total of 2389 reflections were measured and 2345 were independent. Final $R 1=0.0479, w R 2=0.1325(2389$ references; $I>2 \sigma(I)$, and $\mathrm{GOF}=1.080$ (for all data, $R 1=0.0483$, $w R 2=0.1333)$.

\section{(2R,5R,6S,4'R)-6-[1'-(tert-Butyldimethylosiloxy)ethyl]-2-}

\section{methoxycarbonyl-1-azabicyclo[3.2.0] heptan-3,7-di-one (20)}

To a solution of compound 19 (84 mg, $0,24 \mathrm{mmol})$ in $\mathrm{CH}_{2} \mathrm{Cl}_{2}(10 \mathrm{ml})$, $\mathrm{NaHCO}_{3}(81 \mathrm{mg}, 0.96 \mathrm{mmol})$ and Dess-Martin periodinane $(0.20 \mathrm{~g}, 0.48$ $\mathrm{mmol}$ ) were added. The reaction mixture was stirred at room temperature for $40 \mathrm{~min}$. Subsequently, saturated aqueous solution of $\mathrm{Na}_{2} \mathrm{~S}_{2} \mathrm{O}_{3}$ was added. The aqueous phase was extracted with $\mathrm{CH}_{2} \mathrm{Cl}_{2}(3 \times)$. The combined organic extracts were washed with brine, dried over $\mathrm{MgSO}_{4}$ and evaporated. The product was purified on a silica gel column with hexane-EtOAc, 4:6 (v/v) as the eluent to afford 20 (40 mg, $0.11 \mathrm{mmol})$ in $48 \%$ yield.

Colorless crystals m.p. $151-154^{\circ} \mathrm{C} ;[\alpha]_{\mathrm{D}}+125,5\left(\right.$ c $\left.1, \mathrm{CHCl}_{3}\right)$;

IR (film) v: 1256, 1770, 2856, $2929 \mathrm{~cm}^{-1}$;

${ }^{1} \mathrm{H} \mathrm{NMR}\left(600 \mathrm{MHz}, \mathrm{CDCl}_{3}\right) \delta: 0.08\left(\mathrm{~s}, 6 \mathrm{H}, \mathrm{Si}\left(\mathrm{CH}_{3}\right)_{2}\right), 0,90(\mathrm{~s}, 9 \mathrm{H}, t-\mathrm{Bu})$, $1.26\left(\mathrm{~d}, J=6.2 \mathrm{~Hz}, 3 \mathrm{H}, \mathrm{CH}_{3}\right), 2.40(\mathrm{dd}, J=7.7 \mathrm{~Hz}, 18,8 \mathrm{~Hz}, 1 \mathrm{H}, \mathrm{H}-4), 2.86$ (dd, $J=6.9 \mathrm{~Hz}, 18.8 \mathrm{~Hz}, 1 \mathrm{H}, \mathrm{H}-4$ ), 3.10 (dd, $J=2.0 \mathrm{~Hz}, 5,1 \mathrm{~Hz}, 1 \mathrm{H}, \mathrm{H}-6), 3.75$ (s, $3 \mathrm{H}, \mathrm{CO}_{2} \mathrm{CH}_{3}$ ), $4.11-4.15$ (m, 1H, H-5), 4.30 (dq, $J=6.15 \mathrm{~Hz}, 1 \mathrm{H}, \mathrm{CHOSi}$ ), 4.64 (s, 1H, H-2);

${ }^{13} \mathrm{C}$ NMR $\left(150 \mathrm{MHz}, \mathrm{CDCl}_{3}\right) \delta:-5.09,-4.25,17.88,22.59,25.58,27.14$, $41.17,46,87,50.98,53.02,63.93,65.35,68.76,165.46,172.47,207.32$. 
HR MS(ESI) $m / z$ [M+Na] $]^{+}$calculated. for $\mathrm{C}_{16} \mathrm{H}_{27} \mathrm{NO}_{5} \mathrm{SiNa}$ : 364.1556; found: 364.1557

(5R,6S,1'R)-Methyl 3-[(2-acetamidoetyl)thio]-6-[1'-(tertbutyldimethylsiloxy)ethyl)-7-oxo-1-azabicyclo[3.2.0]hept-2-ene-2carboxylate (21)

Compound $20(14 \mathrm{mg}, 0.04 \mathrm{mmol})$ in acetonitrile $(2 \mathrm{ml})$ was cooled to $0{ }^{\circ} \mathrm{C}$ and treated with $\mathrm{N}, \mathrm{N}$-diisopropylethylamine $(8 \mu \mathrm{l}, 0.05 \mathrm{mmol})$, diethyl chlorophosphate $(7 \mu \mathrm{l}, 0.05 \mathrm{mmol})$ and catalytic amount of DMAP. The mixture was stirred for $1 \mathrm{~h}$ at room temperature. Subsequently it was cooled to $0^{\circ} \mathrm{C}$ and treated with a another portion of $\mathrm{N}, \mathrm{N}$-diisopropylethylamine $(11 \mu \mathrm{l}, 0.06$ mmol) and $N$-acetylcysteamine $(6,5 \mu \mathrm{l}, 0.06 \mathrm{mmol})$. The mixture was stirred overnight at room temperature. Subsequently it was evaporated under reduced pressure and purified on a silica gel column using toluene-i-propanol, 4:1(v/v) as an eluent to afford $21(8 \mathrm{mg}, 0.018 \mathrm{mmol})$ in $44 \%$ yield.

Easily solidifing syrup; $[\alpha]_{\mathrm{D}}+52,3\left(c 0.69, \mathrm{CHCl}_{3}\right)$; IR (film) v: 1659, 1748, 1758, 2898, 2929, $2953 \mathrm{~cm}^{-1}$;

${ }^{1} \mathrm{H}$ NMR $\left(600 \mathrm{MHz}, \mathrm{CDCl}_{3}\right) \delta: 0.06\left(\mathrm{~s}, 6 \mathrm{H}, \mathrm{Si}\left(\mathrm{CH}_{3}\right)_{2}\right), 0,90(\mathrm{~s}, 9 \mathrm{H}, t-\mathrm{Bu})$, $1.24\left(\mathrm{~d}, J=6.1 \mathrm{~Hz}, 3 \mathrm{H}, \mathrm{CH}_{3}\right), 1.97\left(\mathrm{~s}, 3 \mathrm{H}, \mathrm{CH}_{3}\right), 2.88-2.94(\mathrm{~m}, 1 \mathrm{H}, \mathrm{SCH})$, 2.99-3.06 (m, 2H, SCHH, H-4), 3.10 (dd, $J=2.7 \mathrm{~Hz}, 6,1 \mathrm{~Hz}, 1 \mathrm{H}, \mathrm{H} 6$ ), 3.22-3.29 (m, $1 \mathrm{H}, \mathrm{H}-4), 3.39-3.49$ (m, $2 \mathrm{H}, \mathrm{CHHNHAc}), 3.81\left(\mathrm{~s}, 3 \mathrm{H}, \mathrm{CO}_{2} \mathrm{CH}_{3}\right.$ ), 4.14-4.21 (m, 2H, H5, CHOSi), 5.96 (bs, 1H, NHAc);

${ }^{13} \mathrm{C}$ NMR $\left(150 \mathrm{MHz}, \mathrm{CDCl}_{3}\right) \delta:-4.93,-4.26,17.94,22.51,23.17,25.62$, $25.68,31.87,39.70,40.10,52.16,52.64,66.23,67.38,124.92,145.93,161.90$, 170.44, 175.98.

HR MS(ESI) $m / z[\mathrm{M}+\mathrm{Na}]^{+}$calculated. for $\mathrm{C}_{20} \mathrm{H}_{34} \mathrm{~N}_{2} \mathrm{O}_{5} \mathrm{SNaSi}$ : 465.1855; found: 465.1858

\section{$(3 S, 4 R, 1 ' R)-4-(2$ '-acetamidoethyltiocarbonylmethyl)-3-[1'-(tert- butylodimethylsiloxy) ethyl]-azetidin-2-one (22)}

Compound $20(45 \mathrm{mg}, 0,13 \mathrm{mmol})$ in acetonitrile $(2 \mathrm{ml})$ was cooled to $0{ }^{\circ} \mathrm{C}$ and treated with $\mathrm{N}, \mathrm{N}$-diisopropylethylamine $(25 \mu \mathrm{l}, 0.14 \mathrm{mmol})$, diphenyl chlorophosphate $(30 \mu \mathrm{l}, 0.14 \mathrm{mmol})$ and a catalytic amount of DMAP. After $1 \mathrm{~h}$ an another portion of $N, N$-diisopropylethyloamine $(25 \mu \mathrm{l}, 0.14 \mathrm{mmol})$ and $\mathrm{N}$-acetylcysteamine $(15 \mu \mathrm{l}, 0.14 \mathrm{mmol})$ were added. The mixture was stirred overnight. Subsequently it was evaporated under reduced pressure and the residue was purified by chromatography using hexane-acetone, 4:6 (v/v) as an eluent to give $22(8 \mathrm{mg}, 0.017 \mathrm{mmol})$ in $13 \%$ yield.

Syrup; $[\alpha]_{\mathrm{D}}+12,3\left(c 0,6, \mathrm{CH}_{2} \mathrm{Cl}_{2}\right)$; IR (film) v: 1660, 1682, 1748 2930, 2954, $3202 \mathrm{~cm}^{-1} ;{ }^{1} \mathrm{H}$ NMR $\left(600 \mathrm{MHz}, \mathrm{C}_{6} \mathrm{D}_{6}\right) \delta:-0.01\left(\mathrm{~s}, 3 \mathrm{H}, \mathrm{Si}\left(\mathrm{CH}_{3}\right)_{2}\right), 0.08(\mathrm{~s}, 3 \mathrm{H}$, $\left.\mathrm{Si}\left(\mathrm{CH}_{3}\right)_{2}\right), 0,88$ (s, 9H, $\left.t-\mathrm{Bu}\right), 1.07\left(\mathrm{~d}, J=6.1 \mathrm{~Hz}, 3 \mathrm{H}, \mathrm{CH}_{3}\right), 1.47(\mathrm{~s}, 3 \mathrm{H}, \mathrm{Ac})$, $2.56(\mathrm{dd}, J=2.4 \mathrm{~Hz}, 6,1 \mathrm{~Hz}, 1 \mathrm{H}, \mathrm{H}-3), 2.58-2.73\left(\mathrm{~m}, 4 \mathrm{H}, \mathrm{SCH}_{2}, \mathrm{CH}_{2} \mathrm{COS}\right.$ ), 3.03-3.12 (m, $\left.2 \mathrm{H}, \mathrm{CH}_{2} \mathrm{NH}\right), 3.19\left(\mathrm{~s}, 3 \mathrm{H}, \mathrm{CO}_{2} \mathrm{CH}_{3}\right), 3.89\left(\mathrm{~m}, 2 \mathrm{H}, \mathrm{NCH}_{2} \mathrm{CO}_{2}\right.$ ), 4.04-4.10 (m, $1 \mathrm{H}, \mathrm{CHOSi}), 4.10-4.14(\mathrm{~m}, 1 \mathrm{H}, \mathrm{H}-4), 4.72$ (bs, $1 \mathrm{H}, \mathrm{NH})$; ${ }^{13} \mathrm{C} \mathrm{NMR}\left(150 \mathrm{MHz}, \mathrm{CDCl}_{3}\right) \delta:-5.22,-4.63,22.28,22.30,25.58,28.60,38.70$, 42.20, 47.61, 51.32, 52.13, 63.71, 66.03, 128.05, 166.53, 168.53, 168.88, 197.08.

HR MS(ESI) $m / z[\mathrm{M}+\mathrm{Na}]^{+}$calculated for $\mathrm{C}_{20} \mathrm{H}_{36} \mathrm{~N}_{2} \mathrm{O}_{6} \mathrm{SiNaS}$ : 483.1961 ; found: 483.1958 .

\section{CONFLICT OF INTEREST}

The authors declare no conflict of interest.

\section{ACKNOWLEDGEMENTS}

Financial support by the European Union within European Regional Development Fund, Project POIG.01.01.02.-14-102/09 is gratefully acknowledged. MS thanks the National Science Centre for Preludium grant UMO-2011/03/N/ST5/04435.
1 Coulton, S. \& Hunt, E. in Progress in Medicinal Chemistry (eds Ellis G. P. \& Luscombe D. K.) 99-145 (Elsevier, Cambridge, UK, 1996).

2 Hashizume, T. \& Morishima, H. Design and synthesis of new 1-betamethylcarbapenems. Drugs of the Future 25, 833-841 (2000).

3 Morin, R. B. \& Gorman, M. Chemistry and Biology of $\beta$-Lactam Antibiotics (Academic Press, New York, USA, 1982).

4 Bouffard, F. A. Thienamycin Total Synthesis. 1. Synthesis of azetidinone precursors of $( \pm)$-thienamycin and its stereoisomers. J. Org. Chem. 45, 1130-1135 (1980).

5 McCormick, J. P. \& Thomason, T. Total synthesis of thienamycin. J. Am. Chem. Soc. 100, 313-315 (1978).

6 Kametani, T., Huang, S.-P., Yokohama, S., Suzuki, Y. \& Ihara, M. Studies on the syntheses of heterocyclic compounds. A formal total synthesis of $( \pm)$-thienamycin and a ( \pm )-decysteaminylthienamycin derivative. J. Am. Chem. Soc. 102, 2060-2065 (1980).

7 Schmitt, S. M., Johnston, D. B. R. \& Christensen, B. G. Thienamycin total synthesis. 2. Model studies-synthesis of a simple 2- (alkylthio) carbapen-2-em. J. Org. Chem. 45, 1135-1142 (1980).

8 Schmitt, S. M., Johnston, D. B. R. \& Christensen, B. G. Thienamycin total synthesis. 3. Total synthesis of $( \pm)$-thienamycin and $( \pm)$-8-epithienamycin. J. Org. Chem. 45, 1142-1148 (1980).

9 Wentrup, C. \& Winter, H.-W. A stereocontrolled synthesis of (+)-thienamycin. J. Am. Chem. Soc. 102, 6161-6163 (1980).

10 Shibasaki, M., Nishida, A. \& Ikegami, S. A. Mild method for the conversion of propiolic ester to $\beta$-keto esters. Application to the formal synthesis of $( \pm)$-thienamycin. Tetrahedron Lett. 23, 2875-2878 (1982).

11 Shibasaki, M., Nishida, A. \& Ikegami, S. A. A Simple preparation of (+) -4phenylthioazetidin-2-one and an asymmetric Synthesis of (+)-thienamycin. J. Chem. Soc. Chem. Commun. 1324-1325 (1982).

12 Tatsuta, K., Takahashi, M., Tanaka, N. \& Chikauchi, K. Novel synthesis of (+)-4acetoxy-3-hydroxyethyl-2-azetidinone from carbohydrate. Total synthesis of (+)-thienamycin. J. Antibiot. 53, 1231-1234 (2000).

13 Chida, N. \& Sato, T. in Comprehensive Chirality Vol. 2 (eds Carreira E. M. \& Yamamoto H.) 207-239 (Elsevier, Amsterdam, 2012).

14 Khangarot, R. K. \& Kaliappan, K. P. A stereoselective synthesis of sugar-derived chiral $\beta$-lactams Eur. J. Org. Chem. 30, 6117-6127 (2011).

15 Desvergnes, S., Py, S. \& Valle'e, Y. Total synthesis of (+)-hyacinthacine A2 based on Sml2-induced nitrone umpolung. J. Org. Chem. 70, 1459-1462 (2005).

16 Ludek, O. R. \& Marquez, V. E. A greener enantioselective synthesis of the antiviral agent Northmethanocarbathymidine (N-MCT) from 2-deoxy-D-ribose. Tetrahedron 65, 8461-8467 (2009).

17 Wang, W.-B. et al. A practical synthesis of sugar-derived cyclic nitrones: Powerful synthons for the synthesis of iminosugars. Synlett. 3, 488-492 (2010).

18 Maciejko, M. et al. An entry to carbapenem antibiotics scaffold via asymmetric Kinugasa reaction. Synthesis 44, 2825-2839 (2012).

19 Yoshida, M., Ohsawa, Y. \& Ihara, M. Palladium-catalyzed carbon dioxide eliminationfixation reaction of 4-methoxycarbonyloxy-2-buten-1-ols. J. Org. Chem. 69, 1590-1597 (2004).

20 Santos, D., Ariza, X., Garcia, J. \& Sónchez, C. A new synthetic approach to the lactol moiety of halichoblelide. Tetrahedron 67, 5184-5188 (2011).

21 Priebe, W. et al. D-Glucose and D-mannose-based metabolic probes. Part 3: Synthesis of specifically deuterated D-glucose, D-mannose, and 2-deoxy-D-glucose. Carbohydr. Res. 368, 111-119 (2013).

22 Kovóč, P. \& Petríkovó, M. Alternative syntheses of methylated sugars. V. Methyl furanosides of 6-O- and 2,6-Di-O-methyl-D-glucose. Chem. Zvesti. 26, 71-75 (1972).

23 Desvergnes, S., Vallée, Y. \& Py, S. Novel polyhydroxylated cyclic nitrones and $\mathrm{N}$-hydroxypyrrolidines through BCl3-mediated deprotection. Org. Lett. 10, 2967-2970 (2008).

24 Holmes, A. B. et al. Selective cleavage of benzyl ether. Synlett. 663-664 (1993).

25 Dess, D. B. \& Martin, J. C. Readily accessible 12-I-5 oxidant for the conversion of primary and secondary alcohols to aldehydes and ketones. J. Org. Chem. 48, 4155-4156 (1983).

26 Bal, B. S., Childers, W. E. Jr \& Pinnick, H. W. Oxidation of $\alpha, \beta$-unsaturated aldehydes. Tetrahedron 37, 2091-2096 (1981).

27 Nagasawa, T., Nukada, T. \& Kuwahara, S. Synthesis of aspergillide A via prolinecatalyzed trans-to-cis isomerization of a substituted tetrahydropyran. Tetrahedron 67, 2882-2888 (2011)

28 Arndt, F. in Organic Synthesis Coll Vol. II (ed. Blatt, A. H.) 165-167 (John Wiley \& sons, New York, 1943).

29 Hanessian, S., Desilets, D. \& Bennani, L. Y. A novel ring-closure strategy for the carbapenems: the total synthesis of (+)-thienamycin. J. Org. Chem. 55, 3098-3103 (1990). 